\title{
Communication
}

\section{Subgridding Boundary Conditions to Model Arbitrarily Dispersive Thin Planar Materials}

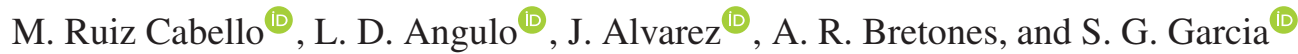

\begin{abstract}
In a previous work, we presented a hybrid implicitexplicit Crank-Nicolson finite-difference time-domain method for treating multilayered lossy thin slabs. The main advantage of this method was its capability to overcome certain late-time stability issues of the conventional surface impedance boundary condition approaches. In this communication, we extend this method to deal with thin slabs having arbitrarily dispersive profiles. This approach is validated with the analysis of a spherical shell made of a metallic wire mesh whose macroscopic equivalent constitutive parameters are derived from its microscopic structure. The results for the electric field inside the sphere are compared against the analytical data and show good agreement with them.
\end{abstract}

Index Terms-Finite differences, subcell models, thin-layer modeling, time domain.

\section{INTRODUCTION}

Some of the modern materials employed in aeronautic and automotive industries consist of multilayered carbon-fiber composite (CFC) panels, highly valued for their mechanical properties and lightweight. However, the electrical conductivity of carbon fiber is significantly lower than that of most metals. Consequently, their shielding effectiveness (SE) against electromagnetic (EM) fields and lightning strikes is reduced. For this reason, manufacturers usually include conductive layers of metallic meshes, foils, honeycomb structures, and so on [1]. A common characteristic of these materials is that they are often electrically thin and present complex frequency-dependent profiles of their scattering parameters (S-parameters) [2]-[10].

Simulating these thin panels directly with the finite-difference time-domain (FDTD) method [11], [12] would require very small discretization steps to resolve the geometrical details and would be computationally prohibitive. A more efficient approach is to use subcell techniques that replace these thin slabs by an equivalent model, which is subsequently coupled with the usual 3-D-Yee FDTD method. One of the most successful methods using this approach is the network impedance boundary conditions (NIBCs) method [13], which takes into account wave penetration at each side of the material, in contrast to the one-sided classical surface impedance boundary condition (SIBC) [14]. NIBC expresses the tangential $E$-field in terms of the tangential $H$-field on each surface of the slab; these

Manuscript received October 19, 2017; revised May 21, 2018; accepted July 9, 2018. Date of publication August 1, 2018; date of current version October 29, 2018. This work was supported in part by Spanish MINECO, EU FEDER, under Project TEC2013-48414-C3-01, Project TEC2016-79214-C3-3-R, and Project TEC2015-68766-REDC, in part by J. de Andalucia, Spain, under Grant P12-TIC-1442, in part by AIRBUS DS through Alhambra-UGRFDTD, and in part by the CSIRC alhambra.ugr.es Supercomputing Center. (Corresponding author: M. Ruiz Cabello.)

M. R. Cabello, L. D. Angulo, A. R. Bretones, and S. G. Garcia are with the Department of Electromagnetism, University of Granada, 18071 Granada, Spain (e-mail: mcabello@ugr.es; lmdiazangulo@ugr.es; arubio@ugr.es; salva@ugr.es).

J. Alvarez is with Airbus Defense and Space, 28906 Getafe, Spain (e-mail: jesus.g.alvarez@airbus.com).

Color versions of one or more of the figures in this communication are available online at http://ieeexplore.ieee.org.

Digital Object Identifier 10.1109/TAP.2018.2862241

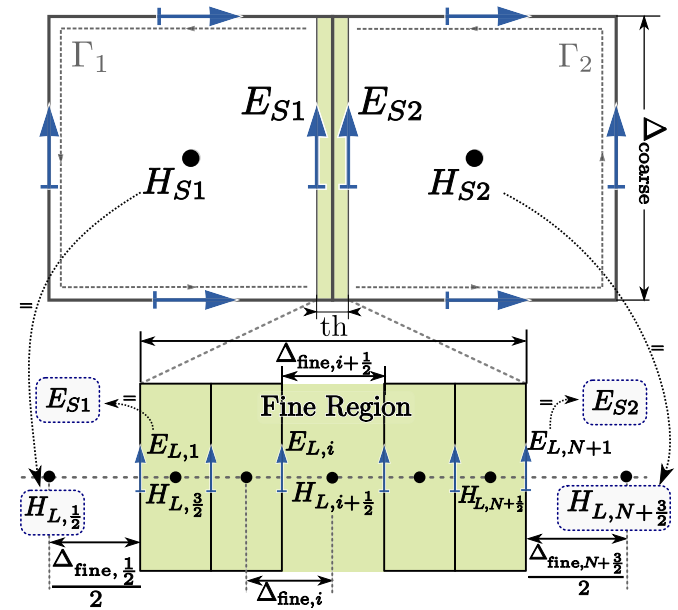

Fig. 1. SGBC boundary: cross section of a thin slab of thickness th located between two FDTD cells (top); subgridding of the slab (bottom).

are related by frequency-dependent impedance relationships, which are translated into the time domain. To make use of NIBC in FDTD, the slab is placed at the tangential $E$-field planes of the 3-D-Yee FDTD arrangement. Since the $E-H$ impedance relationships must be colocated in time and space, an extrapolation from the 3-D-Yee FDTD staggered values is required. This fact has been often reported as a possible cause of the late-time instabilities observed in NIBC simulations [15], [16].

To overcome these instabilities, we developed a new approach, the subgridding boundary condition (SGBC) [17], [18], with the property of preserving the usual Yee positioning of the field components, which eliminates the requirement of performing field extrapolations. Moreover, instead of finding an $\mathrm{E}-\mathrm{H}$ relationship by means of impedance equations, the EM fields are explicitly propagated inside the slab using a 1-D-Yee FDTD scheme. The mesh of this scheme is much finer along its normal direction than the one of the surrounding media (see Fig. 1).

Two approaches were explored with the aim of making SGBC computationally efficient [18]. Initially, an exponential time differencing (ETD) 1-D-Yee FDTD method was used inside the slab, allowing a less restrictive Courant-Friedrichs-Lewy (CFL) stability condition for the time step. An alternative method was considered, consisting in the replacement of the explicit 1-D-Yee FDTD propagator inside the slab by an unconditionally explicit approach based on the 1-D Crank-Nicolson TimeDomain (CNTD) method. The latter was combined with a hybrid implicit-explicit (HIE) algorithm, which naturally connected the 1-D CNTD region to the 3-D-Yee FDTD region, improving the stability properties of the combined scheme.

SGBC was originally formulated assuming that the conductivity, permittivity, and permeability of every single layer comprising the thin slab are known. However, most materials of interest 
do not present a simply ohmic behavior. In this communication, we present an extension of the method described in [17], which allows handling arbitrary frequency-dispersive materials. For this, the 1-D-CNTD and HIE methods are extended by using a piecewise linear recursive convolution (PLRC) method. To test the validity of the new approach, the field penetration inside a hollow spherical shell made of a metallic wire mesh has been calculated using this new method and compared with an analytical method.

\section{SGBC FUNDAMENTALS}

Like the NIBC method, SGBC assumes that waves inside a lossy slab propagate normally to its faces. It can be demonstrated that this is a good approach regardless of the actual angle of incidence, as illustrated by the following example. Assume a lossy slab embedded in free space and the worst case angle of incidence $\theta_{i} \rightarrow \pi / 2$; then, the refracted angle can be proven using Snell's law to be $\left|\theta_{t}\right|<10^{-2}$ if $f[\mathrm{GHz}]<1.8 \sigma[\mathrm{kS} / \mathrm{m}]$ [17]. The SGBC formalism starts considering a lossy thin slab of thickness $t h$. The slab is meshed with a $\Delta_{\text {fine, } i}(1 \leq i \leq N)$ cell size in the direction perpendicular to its interfaces allowing the use of a coarse grid of size $\Delta_{\text {coarse }}$ in the surrounding space, which will be here assumed to be free space (see Fig. 1).

Both NIBC and SGBC duplicate the tangential $E$-fields on the thin-panel surface $E_{S 1}$ and $E_{S 2}$ to account for each face value (see Fig. 1). The $E$-fields $E_{L, i}^{n}$ inside and on the slab $(1 \leq i \leq N+1)$ are located at the usual staggered space-time indexes of the Yee-FDTD cell, with $E_{L, 1}^{n}=E_{S 1}^{n}$ and $E_{L, N+1}^{n}=E_{S 2}^{n}$. The $H$-fields inside the slab $H_{L, i}^{n}$ are staggered by half a space step of the $E$-fields, either colocated at the same time step if using CNTD, or staggered as in the usual Yee FDTD if using ETD. Finally, the outer $H$-fields, laying in the 3-D-Yee FDTD coarse region, are located at the center of the adjacent 3-D cells $H_{S 1}$ and $H_{S 2}$. The SGBC algorithm can then be summarized as follows.

1) The $E$-fields in the fine domain are updated by the 1-D CNTD [17] or by 1-D ETD as in [18].

2) The $H$-fields at the adjacent cells $H_{S 1}^{n+1 / 2}$ and $H_{S 2}^{n+1 / 2}$ employ a discrete form of Ampère's integral law, using $E_{S 1}^{n}$ and $E_{S 2}^{n}$ and taking into account the slab thickness

$$
\begin{aligned}
& H_{S 1}^{n+1 / 2}=H_{S 1}^{n-1 / 2}-\frac{\Delta t}{\mu_{0} A_{1}}\left(\Gamma_{1}^{n}+\Delta_{\text {coarse }} E_{S 1}^{n}\right) \\
& H_{S 2}^{n+1 / 2}=H_{S 2}^{n-1 / 2}-\frac{\Delta t}{\mu_{0} A_{2}}\left(\Gamma_{2}^{n}-\Delta_{\text {coarse }} E_{S 2}^{n}\right)
\end{aligned}
$$

where $\Gamma_{j}^{n}=\sum_{\text {cell } j} \Delta_{\text {coarse }} E_{i}^{n}$ is the usual discrete line integral of the $E$-field components along the three nonboundary edges (for simplicity all assumed to be of size $\Delta_{\text {coarse }}$ ); $A_{1}=A_{2}=$ $\Delta_{\text {coarse }}^{2}$ are the surfaces used for the flux of the $H$-field. It should be noted that $\Gamma_{j}^{n}$ and the surfaces used for the flux $A_{1}$ and $A_{2}$ could be corrected to account for the slab thickness; however, in our experience, this correction has no significant impact on the results.

3) The $E$ - and $H$-fields outside the thin panel $\vec{E}^{n}$ and $\vec{H}^{n+1 / 2}$ are advanced in the usual 3-D Yee-FDTD manner.

It can be demonstrated that ETD-SGBC [19], [20] requires a reduction of around $50 \%$ in the time step to achieve numerical stability if space is sampled using just 15 cells/wavelength at the maximum frequency of interest, whereas reductions of $70 \%$ are required for resolutions of 20 cells/wavelength, as shown in Fig. 2. However, the CNTD-SGBC method does not require reductions in the CFL stability limit, which depends only on the coarse space step, regardless of the fine space step. On the other hand, CNTD requires the solution of a tridiagonal system of equations to find the $E$-fields at each time step. This proves computationally affordable even if a high

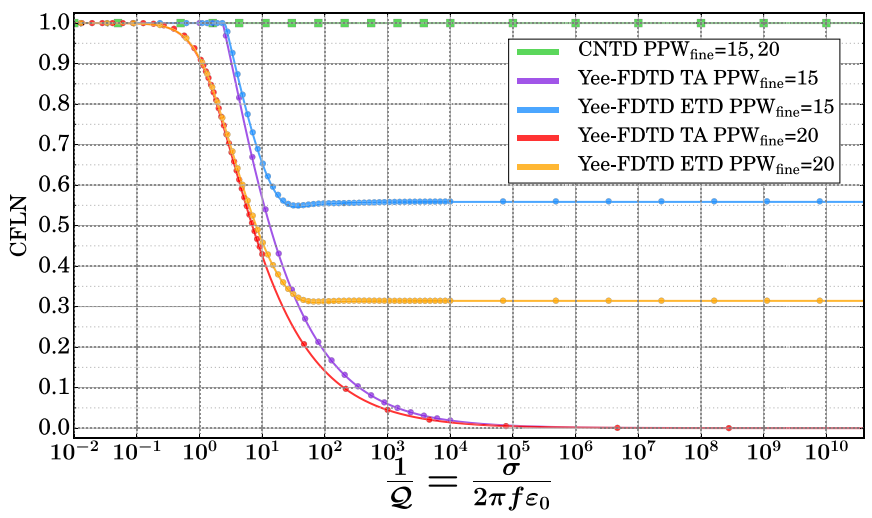

Fig. 2. Comparison between heuristic and theoretical CFL number (CFLN $=$ $c \Delta t / \Delta_{\text {Coarse }}$ ) for a fixed resolution [points per wavelength (PPW)] outside the slab $\mathrm{PPW}_{\text {Coarse }}=20$, and two different resolutions inside the slab $\mathrm{PPW}_{\text {Fine }}=$ 15 , and 20, as a function of the loss tangent of the slab $(1 / Q)$. A 1-D case has been considered. Both analytical (solid lines) and heuristic (dots) numerical results are shown for time average (TA), ETD, and CNTD.

resolution inside the slab is requested. It bears mentioning that both variants of SGBC, ETD, and CNTD, have exhibited a strong latetime stability, whereas NIBC has failed to remain stable, especially in complex resonant problems [20].

\section{Convolutional SGBC CNTD}

The macroscopic EM behavior of a linear isotropic dispersive material can be formulated analytically, in the frequency domain, in terms of its complex permittivity $\varepsilon(\omega)$ and permeability $\mu(\omega)$, relating the electric and magnetic flux densities $\mathcal{D}$ and $\mathcal{B}$ to the fields $E$ and $H[21]$ as

$$
\mathcal{D}(\omega)=\varepsilon(\omega) E(\omega) \text { and } \mathcal{B}(\omega)=\mu(\omega) H(\omega)
$$

or convolutionally in the time domain by

$$
\mathcal{D}(t)=\varepsilon(t) * E(t) \quad \text { and } \quad \mathcal{B}(t)=\mu(t) * H(t) .
$$

The complex functions $\varepsilon(\omega)$ and $\mu(\omega)$ can be expanded into a series of pole-residue partial fractions, by using the vector fitting (VF) procedures proposed in [22] resulting in an effective permittivity and permeability given by

$$
\begin{gathered}
\varepsilon_{\mathrm{eff}}(\omega)=\varepsilon_{\infty}+\sum_{k=1}^{N_{\varepsilon}} \frac{R_{\varepsilon, k}}{j \omega-p_{\varepsilon, k}} \\
\mu_{\mathrm{eff}}(\omega)=\mu_{\infty}+\sum_{k=1}^{\omega_{0} N_{\mu}} \frac{R_{\mu, k}}{j \omega-p_{\mu, k}}
\end{gathered}
$$

where $\varepsilon_{\infty}$ and $\mu_{\infty}$ are the dielectric and magnetic constants, respectively, at the high-frequency limit; $R_{\varepsilon, k}$ and $p_{\mu, k}$ are the residues and poles, respectively.

Transforming (4) into the time domain, we can rewrite (3) as

$$
\begin{aligned}
& \mathcal{D}(t)=\left(\varepsilon_{\infty} \delta(t)+\sum_{k=1}^{N_{\varepsilon}} R_{\varepsilon, k} e^{p_{\varepsilon, k} t} u(t)\right) * E(t) \\
& \mathcal{B}(t)=\left(\mu_{\infty} \delta(t)+\sum_{k=1}^{N_{\mu}} R_{\mu, k} e^{p_{\mu, k} t} u(t)\right) * H(t)
\end{aligned}
$$

where $\delta(t)$ is the Dirac delta function and $u(t)$ is the Heaviside step function.

It shall be noted that the equations in (5) fulfill the causality requirement since $\varepsilon(t)=0$ and $\mu(t)=0$ for $t<0$. They are also 
guaranteed to be stable since the VF procedure enforces the poles to lie in the left complex semiplane $\operatorname{Re}\left(p_{k}\right) \leq 0$. In general, the poles and residues are either real or pairs of complex conjugate. Therefore, we know beforehand that the final time-domain solution is purely real. Hence, we can either compute (5) by using a complex algebra and just retain the real part (double checking that the imaginary part becomes null) or take profit of the method used in [28] to reduce the computational effort by just computing the real part.

\section{A. 1-D-Yee FDTD Formulation}

Let us consider a dispersive slab, fully characterized by an electric conductivity $\sigma$, magnetic conductivity $\sigma_{m}$, electric permittivity $\varepsilon(\omega)$, and magnetic permeability $\mu(\omega)$. It is divided into $N$ cells of size $\Delta_{\text {fine, } i}(1 \leq i \leq N)$, and the electric and magnetic field components inside the slab are denoted as $E_{L, i}$ and $H_{L, i}$ (see Fig. 1). The arbitrary frequency dispersion can be taken into account, in a general manner, by applying the usual Yee FDTD scheme to 1-D Maxwell curl equations inside the slab

$$
\begin{aligned}
& \frac{\mathcal{D}_{L, i}^{n+1}-\mathcal{D}_{L, i}^{n}}{\Delta t}+\sigma \frac{E_{L, i}^{n+1}+E_{L, i}^{n}}{2}=\frac{H_{L, i-\frac{1}{2}}^{n+\frac{1}{2}}-H_{L, i+\frac{1}{2}}^{n+\frac{1}{2}}}{\Delta_{\text {fine }, i}} \\
& \frac{\mathcal{B}_{L, i+\frac{1}{2}}^{n+\frac{1}{2}}-\mathcal{B}_{L, i+\frac{1}{2}}^{n-\frac{1}{2}}}{\Delta t}+\sigma_{M} \frac{H_{L, i+\frac{1}{2}}^{n+\frac{1}{2}}+H_{L, i+\frac{1}{2}}^{n-\frac{1}{2}}}{2}=\frac{E_{L, i-1}^{n+\frac{1}{2}}-E_{L, i}^{n+\frac{1}{2}}}{\Delta_{\text {fine }, i+\frac{1}{2}}}
\end{aligned}
$$

together with the PLRC algorithm [23] to discretize the constitutive relationships given in (5)

$$
\begin{aligned}
\mathcal{D}_{L, i}^{n} & =\varepsilon_{0} \varepsilon_{r} E_{L, i}^{n}+\sum_{k=1}^{N_{\varepsilon}} \zeta_{\varepsilon, i, k}^{n} \\
\mathcal{B}_{L, i+\frac{1}{2}}^{n+\frac{1}{2}} & =\varepsilon_{0} \varepsilon_{r} H_{L, i+\frac{1}{2}}^{n+\frac{1}{2}}+\sum_{k=1}^{N_{\mu}} \zeta_{\mu, i+\frac{1}{2}, k}^{n+\frac{1}{2}}
\end{aligned}
$$

where $\zeta^{n}$ are assessed by its recursive forms

$$
\begin{aligned}
\zeta_{\varepsilon, i, k}^{n} & =q_{1, \varepsilon, k} E_{L, i}^{n}+q_{2, \varepsilon, k} E_{L, i}^{n-1}+q_{3, \varepsilon, k} \zeta_{\varepsilon, i, k}^{n-1} \\
\zeta_{\mu, i+\frac{1}{2}, k}^{n+\frac{1}{2}} & =q_{1, \mu, k} H_{L, i+\frac{1}{2}}^{n+\frac{1}{2}}+q_{2, \mu, k} H_{L, i+\frac{1}{2}}^{n-\frac{1}{2}}+q_{3, \mu, k} \zeta_{\mu, i+\frac{1}{2}, k}^{n-\frac{1}{2}}
\end{aligned}
$$

and $q$ coefficients in (8) are given in Appendix A.

Substituting (7) into (6), the final expressions for updating $E$ - and $H$-fields can be calculated as

$$
\begin{aligned}
E_{L, i}^{n+1}= & C_{a, i} E_{L, i}^{n}+C_{b, i}\left(H_{L, i-\frac{1}{2}}^{n+\frac{1}{2}}-H_{L, i+\frac{1}{2}}^{n+\frac{1}{2}}\right)+C_{\zeta, i} \zeta_{\varepsilon, i}^{n} \\
H_{L, i+\frac{1}{2}}^{n+\frac{1}{2}}= & D_{a, i+\frac{1}{2}} H_{L, i+\frac{1}{2}}^{n-\frac{1}{2}}+D_{b, i+\frac{1}{2}}\left(E_{L, i}^{n}-E_{L, i+1}^{n}\right) \\
& +D_{\zeta, i+\frac{1}{2}}^{n} \zeta_{\mu, i+\frac{1}{2}}^{n+\frac{1}{2}}
\end{aligned}
$$

where $C, D$, and $\zeta^{n}$ are defined in Appendix B.

It should be noted that the PLRC algorithm achieves the secondorder accuracy, which is similar to that provided by another approach also widely used to analyze the dispersive material, the auxiliary differential equations (ADEs) method [12]. Moreover, both PLRC and ADE lead to comparable computational costs (find a discussion in [23] and [24]).

\section{B. 1-D CNTD Formulation}

The Crank-Nicolson method proposed in [17] for the analysis of lossy thin panels can now be easily modified to deal with an arbitrarily dispersive behavior. We start from the usual 1-D-Yee FDTD scheme given by (9), but now sampling the $H$-field at integer, instead of half-integer, time steps. For this, the $H$-fields in the righthand side (RHS) of (9a) are averaged-in-time, resulting in

$$
\begin{aligned}
E_{L, i}^{n+1}= & C_{a, i} E_{L, i}^{n}+C_{\zeta, i} \zeta_{\varepsilon, i}^{n} \\
& +\frac{1}{2} C_{b, i}\left(H_{L, i-\frac{1}{2}}^{n+1}-H_{L, i+\frac{1}{2}}^{n+1}+H_{L, i-\frac{1}{2}}^{n}-H_{L, i+\frac{1}{2}}^{n}\right) .
\end{aligned}
$$

The $H$-field is assessed by rewriting (9b) at integer time steps

$$
\begin{aligned}
H_{L, i+\frac{1}{2}}^{n+1}=D_{a, i+\frac{1}{2}} H_{L, i+\frac{1}{2}}^{n}+D_{b, i+\frac{1}{2}}\left(E_{L, i}^{n+\frac{1}{2}}\right. & \left.-E_{L, i+1}^{n+\frac{1}{2}}\right) \\
& +D_{\zeta, i+\frac{1}{2} \zeta_{\mu, i+\frac{1}{2}}^{n+1}} .
\end{aligned}
$$

The $E$-fields in the RHS of (11) are also averaged-in-time so that they are sampled at integer time steps. The resulting equation becomes

$$
\begin{aligned}
H_{L, i+\frac{1}{2}}^{n+1}= & D_{a, i+1 / 2} H_{L, i+\frac{1}{2}}^{n}+D_{\zeta, i+\frac{1}{2} \zeta_{\mu, i+\frac{1}{2}}^{n}} \\
& +\frac{1}{2} D_{b, i+1 / 2}\left(E_{L, i}^{n}-E_{L, i+1}^{n}+E_{L, i}^{n+1}-E_{L, i+1}^{n+1}\right) .
\end{aligned}
$$

By extracting $H^{n+1}$ from (12) and inserting it into (10), a tridiagonal system of equations is found for the internal $E$-fields $(i=2, \ldots, N)$

$$
\begin{aligned}
a_{i} E_{L, i-1}^{n+1}+b_{i} E_{L, i}^{n+1}+c_{i} E_{L, i+1}^{n+1}=d_{i}^{n}+C_{\zeta, i} \zeta_{\varepsilon, i}^{n} \\
+\frac{1}{2} C_{b, i}\left(D_{\zeta, i-\frac{1}{2}} \zeta_{\mu, i-\frac{1}{2}}^{n}-D_{\zeta, i+\frac{1}{2}} \zeta_{\mu, i+\frac{1}{2}}^{n}\right)
\end{aligned}
$$

where $a_{i}, b_{i}, c_{i}$, and $d_{i}$ are given in Appendix $\mathrm{C}$, while $C, D$, and $\zeta^{n}$ are defined in Appendix B.

For the $E$-fields on the boundaries $E_{L, 1}^{n+1}$, and $E_{L, N+1}^{n+1}$, a mixed 1-D-CNTD/3-D-Yee FDTD algorithm is implemented to make use of the outer noncolocated 3-D-Yee FDTD $H$-fields $H_{S 1}$ and $H_{S 2}$. Again, we start with (9b) and sample the $H$-field at integer time steps. Subsequently, just the $H$-field components that lie inside the slab are averaged-in-time, resulting in

$$
\begin{aligned}
E_{L, 1}^{n+1}= & C_{a, 1} E_{L, 1}^{n}+C_{b, 1}\left(H_{S 1}^{n+\frac{1}{2}}-\frac{1}{2}\left(H_{L, 3 / 2}^{n}+H_{L, 3 / 2}^{n+1}\right)\right) \\
E_{L, N+1}^{n+1}= & C_{a, N+1} E_{L, N+1}^{n}+C_{b, N+1} \\
& \times\left(\frac{1}{2}\left(H_{L, N+1 / 2}^{n}+H_{L, N+1 / 2}^{n+1}\right)-H_{S 2}^{n+\frac{1}{2}}\right) .
\end{aligned}
$$

Next, the magnetic fields inside the slab $H_{L, 3 / 2}^{n}$ and $H_{L, N+1 / 2}^{n+1}$ found from (12) are inserted into (14), and the following set of equations results:

$$
\begin{aligned}
b_{1} E_{L, 1}^{n+1}+c_{1} E_{L, 2}^{n+1}= & d_{1}^{n}+C_{\zeta, 1} \zeta_{\varepsilon, 1}^{n}-\frac{1}{2} C_{b, 1} D_{\zeta, \frac{3}{2} \zeta_{\mu, \frac{3}{2}}^{n}} \\
a_{N+1} E_{L, N}^{n+1}+b_{N+1} E_{L, N+1}^{n+1}= & d_{N+1}^{n}+C_{\zeta, N+1} \zeta_{\varepsilon, N+1}^{n} \\
& +\frac{1}{2} C_{b, N+1} D_{\zeta, N+\frac{1}{2}} \zeta_{\mu, N+\frac{1}{2}}^{n} .
\end{aligned}
$$

Equations (10) and (15) can be formulated in the specific matrix form given in Appendix C [see (18)]. A back-substitution algorithm enables us to solve the tridiagonal system (18) and to find the $E_{L, i}^{n+1}$ values. Inserting them into (12), the $H$-field components $H_{L, i+(1 / 2)}^{n+1}$ can finally be found in an explicit manner. 
TABLE I

12Th-ORder Vector-Fitted Poles And Residues Used Herein

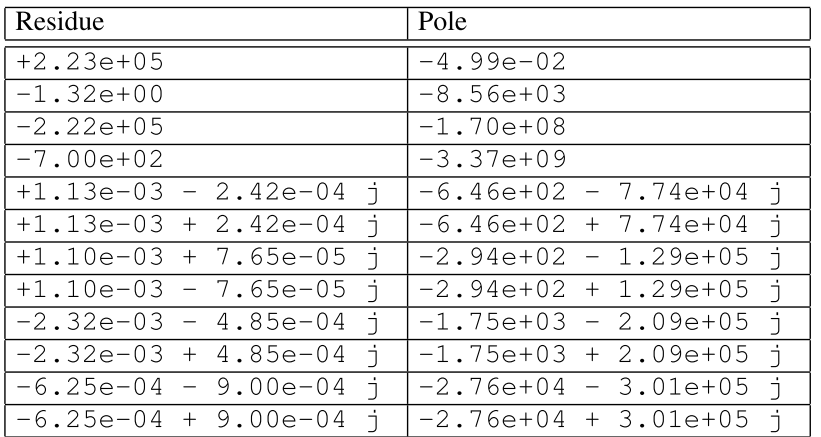
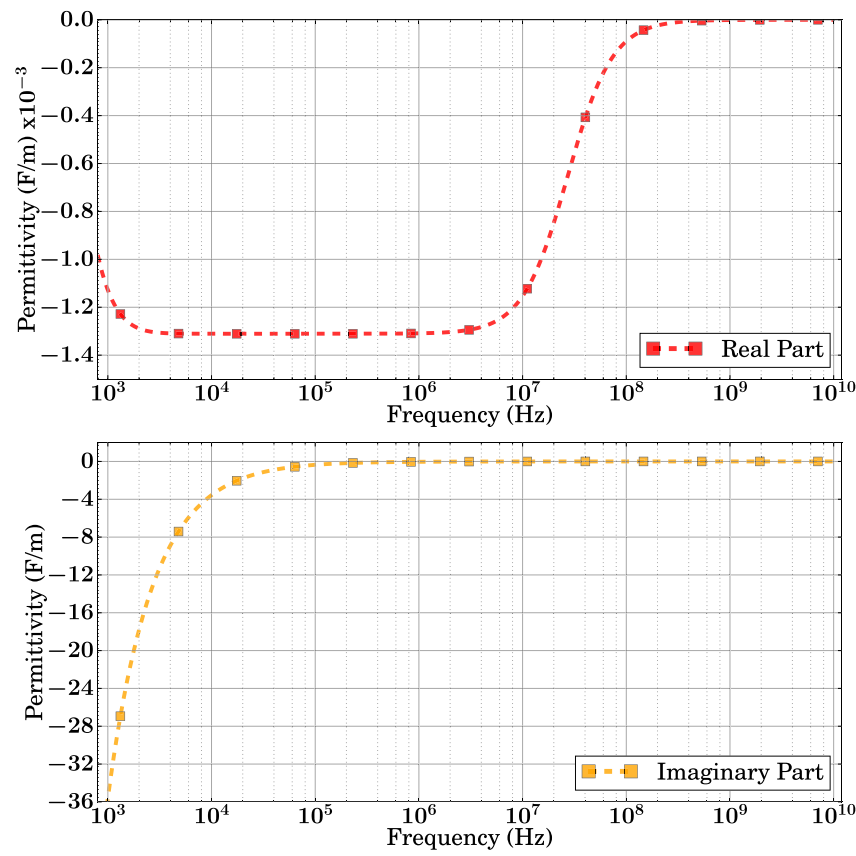

Fig. 3. Frequency behavior of the complex permittivity $\varepsilon=\varepsilon^{\prime}+j \varepsilon^{\prime \prime}$ of the equivalent homogeneous material.

The above-discussed procedure provides a consistent connection between CNTD and 3-D-Yee FDTD, so that the 1-D CNTD employs 3-D-Yee FDTD $H$-fields as source terms, which can be regarded as boundary conditions. This preserves the unconditional stability of the CNTD algorithm and keeps the global time step bounded just by the usual CFL criterion of the 3-D-Yee FDTD coarse zone.

\section{NUMERICAL RESULTS}

A challenging resonant test case based on a spherical shell of thickness $0.1 \mathrm{~mm}$, made of a dispersive material, has been used to validate the proposed method. The average radius of the shell is $1 \mathrm{~m}$, and the shell material microscopically consists of a copper mesh of crossed wires with $50 \mu \mathrm{m}$ diameter each. The macroscopic S-parameters of this material under plane-wave incidence have been found by using the method described in [6]. From these, equivalent constitutive parameters have been established using the parameter-extraction procedures explained in [25]. The permeability results are the same as in free space. The frequency-dependent permittivity has been vector-fitted by the 12th-order model with the values of the residues and poles, which are given in Table I. A plot of its equivalent conductivity versus frequency is shown in Fig. 3.

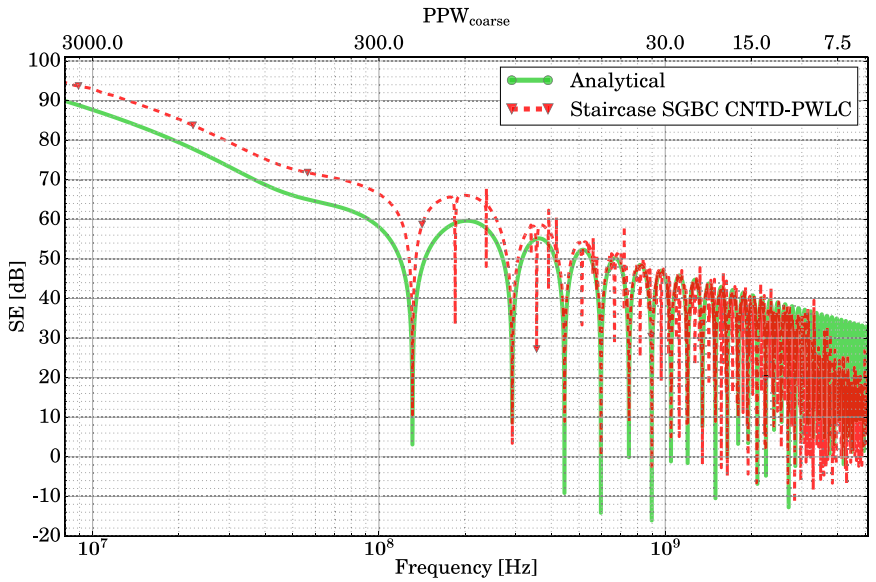

Fig. 4. $E$-field at the center of the sphere. The SE (inverse of the transfer function) has been used as the figure-of-merit defined as $\mathrm{SE}=$ $20 \log \left(\left|E_{\text {incident }}\right| /\left|E_{\text {center }}\right|\right)$.

In this simulation, $\Delta_{\text {coarse }}=10 \mathrm{~mm}$, the $\mathrm{SGBC}$ method employs four cells to sample the spherical shell, which provides a minimum resolution of $100 \mathrm{PPW}$ at $1.5 \mathrm{GHz}$.

The structure is illuminated with a plane wave impinging at $45^{\circ}$ with respect to the $\mathrm{x}$-axis, polarized and propagating in the $x y$ plane. The computational volume is truncated by a convolutional PML. Results for the $E$-field at the center of the sphere are shown in Fig. 4. The results are compared with the closed-form analytical data found from [26]. Good agreement is found between the numerical and theoretical data for frequencies lower than approximately $3 \mathrm{GHz}$, but FDTD results show a nonphysical decreasing trend after that. A likely explanation is that, at frequencies greater than $3 \mathrm{GHz}$, the outer resolution is too low, just $10 \mathrm{PPW}$. Furthermore, the value at $f=3 \mathrm{GHz}$ of the equivalent electric conductivity, established by applying the parameter-extraction procedures, was approximately $\sigma \approx 100 \mathrm{~S} / \mathrm{m}$, which, according to the limit given in Section II, yields a maximum frequency of approximately $0.18 \mathrm{GHz}$ for the TEM propagation hypothesis to be valid. Finally, some spurious spikes are noticed at some frequencies for the FDTD results, which have been demonstrated [27] to be inherent to the staircase approximation made for the curved surface of the sphere.

\section{CONCLUSION}

The SGBC subgridding procedure was introduced in [17], for the FDTD analysis of electrically lossy thin panels, as a stable alternative to the classical NIBC techniques which suffer from late-time instabilities. In this communication, a more general formulation of SGBC has been presented for the modeling of thin panels with arbitrary frequency-dispersive electric and magnetic characteristics. For this aim, the unconditionally stable 1-D CNTD method has been combined with the PLRC formulation and linked to the 3-D-Yee FDTD by a natural HIE scheme. A challenging example of a spherical electrically dispersive shell has been considered. The FDTD-SGBC solution has been compared with the analytic solution showing a very close agreement for frequencies lower than the upper validity limit of the method. Finally, although this communication has employed the PLRC method, the proposed CNTD method can also be formulated with the ADE method of [28] instead. In general, both formulations are equally suitable for modeling the dispersive effect of a material in FDTD methods, and a further discussion can be found in [23] and [24]. 
APPENDIX A

Piecewise Linear Convolution CoefFicients

$$
\begin{aligned}
q_{1, \nu, k} & =\frac{R_{v, k}}{p_{\nu, k}^{2} \Delta t}\left(1+e^{p_{v, k} \Delta t}\left(p_{\nu, k} \Delta t-1\right)\right) \\
q_{2, \nu, k} & =\frac{R_{v, k}}{p_{v, k}^{2} \Delta t}\left(e^{p_{v, k} \Delta t}-p_{\nu, k} \Delta t-1\right) \\
q_{3, \nu, k} & =e^{p_{v, k} \Delta t} \\
q_{1, v} & =\sum_{k=1}^{N_{v}} q_{1, \nu, k} \quad q_{2, v}=\sum_{k=1}^{N_{v}} q_{2, \nu, k}
\end{aligned}
$$

where $v$ denotes both $\varepsilon$ and $\mu$.

$$
\begin{gathered}
\text { FDTD COEFFICIENTS } \\
C_{a}=-\frac{\sigma \Delta t+2\left(q_{2, \varepsilon}-\varepsilon_{0} \varepsilon_{r}\right)}{\sigma \Delta t+2\left(q_{1, \varepsilon}+\varepsilon_{0} \varepsilon_{r}\right)} \\
C_{b}=\frac{2 \Delta t / \Delta_{\text {fine }, i}}{\sigma \Delta t+2\left(q_{1, \varepsilon}+\varepsilon_{0} \varepsilon_{r}\right)} \\
C_{\zeta}=-\frac{2}{\sigma \Delta t+2\left(q_{1, \varepsilon}+\varepsilon_{0} \varepsilon_{r}\right)} \\
\zeta_{\varepsilon, i}^{n}=\sum_{k=1}^{N_{\varepsilon}\left(q_{3, \varepsilon, k}-1\right) \zeta_{\varepsilon, i, k}^{n}} \\
D_{a}=-\frac{\sigma_{M} \Delta t+2\left(q_{2, \mu}-\mu_{0} \mu_{r}\right)}{\sigma_{M} \Delta t+2\left(q_{1, \mu}+\mu_{0} \mu_{r}\right)} \\
D_{b}=\frac{2 \Delta t / \Delta_{\text {fine }, i+\frac{1}{2}}}{\sigma_{M} \Delta t+2\left(q_{1, \mu}+\mu_{0} \mu_{r}\right)} \\
D_{\zeta}=-\frac{2}{\sigma_{M} \Delta t+2\left(q_{1, \mu}+\varepsilon_{0} \mu_{r}\right)} \\
\zeta_{\mu, i}^{n}=\sum_{k=1}^{N_{\mu}\left(q_{3, \mu, k}-1\right) \zeta_{\mu, i, k}^{n} \cdot}
\end{gathered}
$$

\section{APPENDIX C \\ CNTD IN 1-D}

The CNTD approach takes the general form

$$
[M][E]^{n+1}=[d]^{n}+\left[d_{\text {disp }}\right]^{n}
$$

where $[E]^{n+1}$ is a column vector with all the unknowns, $[d]^{n}$ is the independent term, which is a function of previous values of $E$ - and $H$-fields, $\left[d_{\text {disp }}\right]^{n}$ includes the dispersive propagation terms, and $[M]$ is a tridiagonal matrix with constant coefficients

$$
\begin{aligned}
{[M] } & =\left(\begin{array}{cccccc}
b_{1} & c_{1} & 0 & \cdots & \cdots & 0 \\
a_{2} & b_{2} & c_{2} & \ddots & & \vdots \\
0 & a_{i} & b_{i} & c_{i} & \ddots & \vdots \\
\vdots & \ddots & & \ddots & & 0 \\
\vdots & & \ddots & a_{N} & b_{N} & c_{N} \\
0 & \ldots & \ldots & 0 & a_{(N+1)} & b_{(N+1)}
\end{array}\right) \\
{[E]^{n+1}=} & \left(\begin{array}{c}
E_{L, 1}^{n+1} \\
\vdots \\
E_{L, i}^{n+1} \\
\vdots \\
E_{L,(N+1)}^{n+1}
\end{array}\right), \quad[d]^{n}=\left(\begin{array}{c}
d_{1}^{n} \\
\vdots \\
d_{i}^{n} \\
\vdots \\
d_{N+1}^{n}
\end{array}\right)
\end{aligned}
$$

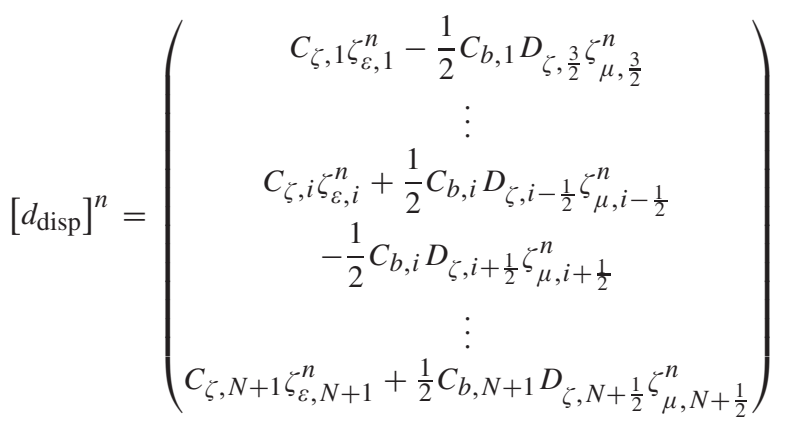

with the subscript $i$ denoting the space position $(i \Delta)$ and the superscript $n$ the time instant $(n \Delta t)$, and with the usual notation for the evolution constants $C_{a}, C_{b}, D_{a}$, and $D_{b}$ [12]. The rest of the terms $\left(a_{i}, b_{i}, c_{i}, d_{i}\right)$ are defined as

$$
\begin{aligned}
a_{i}= & -\frac{1}{4} C_{b, i} D_{b, i-1 / 2} \\
c_{i}= & -\frac{1}{4} C_{b, i} D_{b, i+1 / 2} \\
b_{i}= & \left(1-a_{i}-c_{i}\right) \\
a_{1}= & 0, \quad c_{N+1}=0 \\
d_{i}^{n}= & \frac{1}{2} C_{b, i}\left(1+D_{a, i-1 / 2}\right) H_{L, i-\frac{1}{2}}^{n} \\
& -\frac{1}{2} C_{b, i}\left(1+D_{a, i+1 / 2}\right) H_{L, i+\frac{1}{2}}^{n} \\
& +\frac{1}{4} C_{b, i} D_{b, i-1 / 2} E_{L(i-1)}^{n} \\
& +\left(C_{a, i}-\frac{1}{4} C_{b, i}\left(D_{b, i-1 / 2}+D_{b, i+1 / 2}\right)\right) E_{L, i}^{n} \\
& +\frac{1}{4} C_{b, i} D_{b, i+1 / 2} E_{L, i+1}^{n} \\
d_{1}= & \left(C_{a, 1}-\frac{1}{4} C_{b, 1} D_{b, 3 / 2}\right) E_{L, 1}^{n}+\left(\frac{1}{4} C_{b, 1} D_{b, 3 / 2}\right) E_{L, 2}^{n} \\
& +C_{b, 1}\left(H_{S 1}^{n+\frac{1}{2}}-\frac{1}{2}\left(1+D_{a, 3 / 2}\right) H_{L, 3 / 2}^{n}\right) \\
& \left(C_{a, N+1}-\frac{1}{4} C_{b, N+1} D_{b, N+1 / 2}\right) E_{L, N+1}^{n} \\
& +\frac{1}{4} C_{b, N+1} D_{b, N+1 / 2} E_{L, N}^{n} \\
& +C_{b, N+1}\left(\frac{1}{2}\left(1+D_{a, N+1 / 2}\right) H_{L, N+1 / 2}^{n}-H_{S 2}^{n+\frac{1}{2}}\right) \\
d_{N+1} &
\end{aligned}
$$

It should be noted that the nondispersive method proposed in [17] is recovered when $\left[d_{\text {disp }}\right]^{n}=[0]$.

\section{REFERENCES}

[1] C. Karch and C. Metzner, "Lightning protection of carbon fibre reinforced plastics-An overview," in Proc. 33rd Int. Conf. Lightning Protection (ICLP), Sep. 2016, pp. 1-8.

[2] W. B. Weir, "Automatic measurement of complex dielectric constant and permeability at microwave frequencies," Proc. IEEE, vol. 62, no. 1, pp. 33-36, Jan. 1974.

[3] A. Tamburrano, D. Desideri, A. Maschio, and M. S. Sarto, "Coaxial waveguide methods for shielding effectiveness measurement of planar materials Up to $18 \mathrm{GHz}$," IEEE Trans. Electromagn. Compat., vol. 56, no. 6, pp. 1386-1395, Dec. 2014.

[4] H. Vasquez, L. Espinoza, K. Lozano, H. Foltz, and S. Yang, "Simple device for electromagnetic interference shielding effectiveness measurement," in Proc. EMC IEEE EMC Soc. Newslett., vol. 220, 2009, pp. $62-68$.

[5] M. S. Sarto and A. Tamburrano, "Innovative test method for the shielding effectiveness measurement of conductive thin films in a wide frequency range," IEEE Trans. Electromagn. Compat., vol. 48, no. 2, pp. 331-341, May 2006.

[6] M. S. Sarto, S. Greco, and A. Tamburrano, "Shielding effectiveness of protective metallic wire meshes: EM modeling and validation," IEEE Trans. Electromagn. Compat., vol. 56, no. 3, pp. 615-621, Jun. 2014. 
[7] J. R. Wait, "Reflection at arbitrary incidence from a parallel wire grid," Appl. Sci. Res., A, vol. 4, no. 1, pp. 393-400, 1955

[8] C. L. Holloway, M. S. Sarto, and M. Johansson, "Analyzing carbonfiber composite materials with equivalent-Layer models," IEEE Trans. Electromagn. Compat., vol. 47, no. 4, pp. 833-844, Nov. 2005.

[9] V. Volski and G. A. Vandenbosch, "Full-wave electromagnetic modelling of fabrics and composites," Compos. Sci. Technol., vol. 69, no. 2, pp. 161-168, 2009.

[10] V. Préault, R. Corcolle, L. Daniel, and L. Pichon, "Effective permittivity of shielding composite materials for microwave frequencies," IEEE Trans. Electromagn. Compat., vol. 55, no. 6, pp. 1178-1186, Dec. 2013.

[11] K. Yee, "Numerical solution of initial boundary value problems involving Maxwell's equations in isotropic media," IEEE Trans. Antennas Propag., vol. AP-14, no. 3, pp. 302-307, May 1966.

[12] A. Taflove and S. C. Hagness, Computational Electrodynamics: The Finite-Difference Time-Domain Method. Norwood, MA, USA: Artech House, 2005.

[13] M. S. Sarto, "A new model for the FDTD analysis of the shielding performances of thin composite structures," IEEE Trans. Electromagn. Compat., vol. 41, no. 4, pp. 298-306, Nov. 1999.

[14] J. H. Beggs, R. J. Luebbers, K. S. Yee, and K. S. Kunz, "Finitedifference time-domain implementation of surface impedance boundary conditions," IEEE Trans. Antennas Propag., vol. 40, no. 1, pp. 49-56, Jan. 1992.

[15] G. Kobidze, "Implementation of collocated surface impedance boundary conditions in FDTD," IEEE Trans. Antennas Propag., vol. 58, no. 7, pp. 2394-2403, Jul. 2010.

[16] V. Nayyeri, M. Soleimani, and O. M. Ramahi, "Modeling graphene in the finite-difference time-domain method using a surface boundary condition," IEEE Trans. Antennas Propag., vol. 61, no. 8, pp. 4176-4182, Aug. 2013.

[17] M. R. Cabello et al., "A hybrid Crank-Nicolson FDTD subgridding boundary condition for lossy thin-layer modeling," IEEE Trans. Microw. Theory Techn., vol. 65, no. 5, pp. 1397-1406, May 2017.
[18] M. R. Cabello, L. D. Angulo, A. R. Bretones, R. G. Martin, S. G. Garcia, and J. Alvarez, "A novel subgriding scheme for arbitrarily dispersive thin-layer modeling," in IEEE MTT-S Int. Microw. Symp. Dig., May 2017, pp. 266-268.

[19] M. R. Cabello, "Subcell fdtd techniques for electromagnetic compatibility assessment in aeronautics," Ph.D. dissertation, Dept. Electromagn. Phys. Matter, Univ. Granada, Granada, Spain, 2017.

[20] M. R. Cabello et al., "Siva UAV: A case study for the EMC analysis of composite air vehicles," IEEE Trans. Electromagn. Compat., vol. 59, no. 4, pp. 1103-1113, Aug. 2017.

[21] C. A. Balanis, Engineering Electromagnetics, 2nd ed. Hoboken, NJ, USA: Wiley, 2012.

[22] B. Gustavsen and A. Semlyen, "Rational approximation of frequency domain responses by vector fitting," IEEE Trans. Power Del., vol. 14, no. 3, pp. 1052-1061, Jul. 1999.

[23] D. F. Kelley and R. J. Luebbers, "Piecewise linear recursive convolution for dispersive media using FDTD," IEEE Trans. Antennas Propag., vol. 44, no. 6, pp. 792-797, Jun. 1996.

[24] L. J. Prokopeva, J. D. Borneman, and A. V. Kildishev, "Optical dispersion models for time-domain modeling of metal-dielectric nanostructures," IEEE Trans. Magn., vol. 47, no. 5, pp. 1150-1153, May 2011.

[25] X. Chen, T. M. Grzegorczyk, B.-I. Wu, J. Pacheco, Jr., and J. A. Kong, "Robust method to retrieve the constitutive effective parameters of metamaterials," Phys. Rev. E, Stat. Phys. Plasmas Fluids Relat. Interdiscip. Top., vol. 70, no. 1, p. 016608, 2004.

[26] M. Ansarizadeh, "Calculation of the shielding effectiveness of carbonfiber composite structures," Ph.D. dissertation, Dept. Elect. Comput. Eng., Montreal, QC, Canada, Sep. 2013.

[27] J. F. Dawson et al., "Face centered anisotropic surface impedance boundary conditions in FDTD: Improved performance of staircased mesh for shielding problems," in IEEE MTT-S Int. Microw. Symp. Dig., May 2017, pp. 260-262.

[28] M. Han, R. W. Duttonm, and S. Fan, "Model dispersive media in finite-difference time-domain method with complex-conjugate poleresidue pairs," IEEE Microw. Wireless Compon. Lett., vol. 16, no. 3, pp. 119-121, Mar. 2006. 